\title{
Prioritizing clinical research studies during the COVID-19 pandemic: lessons from New York City
}

\author{
Roy M. Gulick, ${ }^{1}$ Magdalena E. Sobieszczyk, ${ }^{2}$ Donald W. Landry, ${ }^{2}$ and Anthony N. Hollenberg ${ }^{1}$
}

'Department of Medicine, Weill Cornell Medicine, New York, New York, USA. ²Department of Medicine, Columbia University Vagelos College of Physicians and Surgeons, New York, New York, USA.

C oronavirus disease 2019 (COVID-19), caused by SARS-CoV-2, was first recognized in Wuhan, China in December 2019 (1) and rapidly spread through Asia and Europe, with the first US case identified in January 2020. The first New York case was reported in early March 2020 and by the first week in April, the New York Presbyterian Hospital system, including Columbia and Weill Cornell, had close to 2500 in-patients with over 700 patients on ventilators. The standard of care for COVID-19 was supportive care only and with significant morbidity and mortality recognized, there was a desperate need to identify effective treatments. Launching clinical research studies during this public health crisis required communication, collaboration, prioritization, keeping current with rapidly changing evidence, and maintaining high standards of scientific integrity and participant safety.

\section{An emerging crisis without proven treatments}

COVID-19 is a mild illness in more than $80 \%$ of people infected, but about $15 \%$ will require hospitalization and about $5 \%$ intensive care unit support (2). Patients most commonly present with symptoms of a viral respiratory illness, but the infection can provoke an intense immune response that leads to cytokine storm, coagulopathy, respiratory failure, and end-organ disease. Some patients progress to acute respiratory distress syndrome (ARDS) and require prolonged mechanical ventilation, sometimes with associated complications. Early reports from China and Europe documented wide-ranging approaches for COVID-19 aimed at each stage of the illness including antivirals, immunomodulators, and combination strategies, with use of repurposed existing drugs and novel investigational agents, for treatment and prevention (Figure 1).
With a multitude of potential options, how does one pursue the most promising clinical trials? Although they are separate medical schools, Columbia and Weill Cornell share our major affiliated hospital, NewYork-Presbyterian. This structure facilitated communication and collaboration from the beginning, and consequently, the leaders of both Departments of Medicine and Divisions of Infectious Disease set up multidisciplinary committees to evaluate current data to prioritize and recommend initiation of COVID-19 clinical studies. The committees broadly represented the relevant medical disciplines and expertise in clinical trials at each institution. Furthermore, the deliberations and decisions made by each of the committees was communicated across the institutions to ensure that parallel trials were opened and that potentially harmful therapies were not pursued. Both committees received hundreds of study ideas - randomized clinical trials; single-arm pilot studies; expanded-access programs; compassionate-use studies, both multicenter and single-patient emergency use; and retrospective observational studies from a variety of sources - international, US government, pharmaceutical companies, and investigator initiated. At the same time, desperate patients, their families, and providers exerted pressure to use specific therapies and strategies. The support of the leadership of the medical schools and the hospital to focus on the most promising clinical studies across institutions was critical to standardizing approaches and advancing the science in service of our patients.

\section{Testing the most promising drug candidates}

Following an early review of available data, we prioritized studies of the inves-

Conflict of interest: The authors have declared that no conflict of interest exists.

Copyright: (c) 2020, American Society for Clinical Investigation.

Reference information: / Clin Invest. 2020;130(9):4522-4524. https://doi.org/10.1172/JCI142151.

tigational antiviral drug remdesivir, the repurposed drug hydroxychloroquine, the immunomodulator sarilumab, and convalescent plasma. Remdesivir demonstrated in vitro activity against SARS-CoV-2 (3) and a favorable safety record from prior clinical studies of Ebola virus. We pursued industry-sponsored randomized phase III multicenter studies of remdesivir in moderate and severe COVID-19 as well as parallel compassionate-use, and later, expanded-access, programs. Ultimately, we prioritized the randomized controlled clinical trials and contributed efficacy and safety data that demonstrated the clinical benefits of remdesivir in COVID-19 (4). The subsequent US FDA emergency use authorization (EUA) of remdesivir, albeit with a limited supply, posed additional challenges in determining which patients received treatment. To address this issue, we convened an expert group who reviewed the available data and developed a hospital-wide policy for remdesivir use.

Hydroxychloroquine, an FDA-approved drug for malaria and certain autoimmune diseases, demonstrated in vitro activity against SARS-CoV-2 (5) and was readily available. With early clinical trial data from China and Europe (6), no other available COVID-19 treatment, and provider and community pressure, we initially recommended hydroxychloroquine off label for hospitalized patients with COVID-19, along with a commitment to collect our data $(7,8)$. Ultimately, prospective clinical trials failed to demonstrate benefit (9), and the use of hydroxychloroquine and further studies were abandoned.

The cytokine storm associated with severe COVID-19 prompted suggestions for investigation of a host of immunomodulatory drugs - both agonists and antagonists directed at multiple steps of the immune cascade. We focused efforts on an early randomized, controlled clinical trial of sarilumab, an IL-6 inhibitor. Another IL-6 inhibitor, tocilizumab, was available off label and there was some tension 


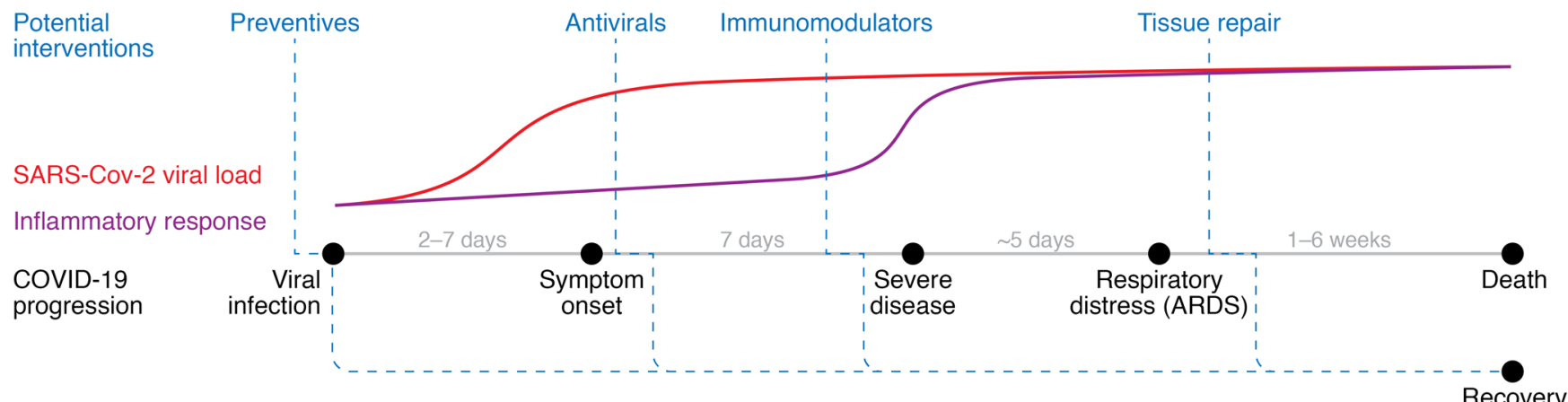

Figure 1. Course of SARS-CoV-2 infection/COVID-19: clinical stages and potential interventions. Displayed pictorially are the clinical stages of disease resulting from SARS-CoV-2 infection and coronavirus infectious disease 2019 (COVID-19). Prior to exposure, preventive strategies may help avoid or abrogate infection. After infection, antivirals may reduce viral replication and resultant complications. If viral replication continues and an inflammatory response develops, immunomodulators could prevent or dampen an exaggerated immune response. If further progression with tissue destruction occurs, cellular therapies could promote tissue repair. Importantly, with novel preventive and therapeutic strategies, recovery from SARS-CoV-2 infection could improve at all stages. Figure adapted with permission from BioCentury (https://www.biocentury.com/trial-timeline).

among patients, their families, providers, and our research staff, between these two approaches; however, after thorough consideration, we chose to prioritize the clinical trial, reserving consideration of the off-label drug only for patients unable or unwilling to participate in the study. Numerous other immunomodulatory drugs including JAK inhibitors and BTK inhibitors were proposed and again, we prioritized multicenter randomized clinical trials over investigatorinitiated pilot studies or single-patient compassionate use.

COVID-19 convalescent plasma posed unique challenges. The strategy of administering convalescent plasma for infectious diseases dates back over 100 years, and this strategy engendered great interest among providers and the community, including the media. A large uncontrolled compassionate-use program of COVID-19 convalescent plasma was instituted, and patients, their families, and providers exerted great pressure for individual patient use, albeit in the absence of demonstrated safety or efficacy. We convened a multidisciplinary group, and guided by the desire to optimize patient safety, prioritized randomized, controlled studies, and developed a hospital-wide policy that we would administer convalescent plasma through this mechanism unless study implementation was impossible, in which case compassionate use was considered. Ultimately, the COVID-19 convalescent plasma compassionate-use program published findings on 20,000 participants, providing safety, but no efficacy, data (10). Prospective randomized controlled studies of convalescent plasma for COVID-19 are ongoing at both our institutions.

\section{Balancing the need for speed and patient safety}

Developing a clinical trials program during an infectious diseases pandemic poses unique challenges. The desire of patients, their families, and providers to do something - even when there are no proven therapies - is strong. The usual deliberate process of IRB approval and clinical trials contract negotiations contrasts the urgency of providing treatment options. To mitigate this, our institutional IRBs convened daily, and the clinical trials offices escalated reviews. An efficiently transmittable infectious disease like COVID-19 that requires extensive personal protective equipment and the routine exclusion of family members from the bedside complicated the informed-consent process and routine study procedures. The desperation for the latest medical knowledge led to urgent release of clinical data in unconventional ways: media reports from Zoom calls, tweets, press releases, and non-peer-reviewed preprints. Wellrespected journals published COVID-19 studies with less than a dozen patients (11, $12)$, articles that were later deemed "not to meet society standards" (6), and articles that were subsequently retracted (13). All the while, politics and media engaged and influenced COVID-19 clinical research and treatment. Additionally, with a multitude of proposed COVID-19 studies, it became abundantly clear that choosing among trials would become a major issue.

\section{Insights for future efforts}

Acknowledging both accomplishments and missteps, what lessons can be learned from this extraordinary time? First and foremost, initiate broad communication and engagement of everyone concerned on a regular and ongoing basis: patients and their families; providers, scientists, and colleagues; research clinicians and staff; research administration at the IRB and contracts offices; medical school and hospital leaders; public affairs; and the media. Second, reach out to colleagues across disciplines to foster new ideas and initiate new collaborations. One example was to engage our transfusion medicine service and blood banks to establish a study to collect convalescent plasma from people who recovered from COVID-19 for later use in randomized clinical trials. We initiated virtual scientific forums and town halls for basic, translational, and clinical researchers, and clinicians across multiple disciplines to come together, exchange knowledge, and promote discussion and plans. Also, we acknowledged and accepted conflict among stakeholders, but relied on our prioritization committees to advance what we thought were the best clinical trial ideas.

Third, use this broad dialog to evaluate and prioritize research plans and institute an "all hands on deck" strategy - engage research laboratories and clinical research units and redeploy research clinicians and staff as needed from other areas. Fourth, keep current with the challenging and rapidly changing field. Standing virtual meetings between the Depart- 
ment of Medicine, Infectious Diseases, and hospital leadership, and conferences facilitate presentation, discussion, and synthesis of the latest available data. Last and most importantly, maintain standards of scientific integrity, ethical study conduct, and participant safety. The urge to try something for a potentially fatal infectious disease is strong and the pressure to use drugs off label or through singlepatient compassionate-use programs is great. However, the only way to prove or disprove the efficacy of candidate strategies and to assess their safety is to conduct well-designed, randomized controlled clinical studies. Remarkably over the last six months, results from such studies already demonstrate the clinical benefits and preliminary safety of the antiviral drug remdesivir $(4,14)$ and the immunomodulator dexamethasone (15), as well as the lack of efficacy of the previously widely used drugs hydroxychloroquine (9) and lopinavir-ritonavir (16). With worldwide clinical research efforts ongoing, further progress can be anticipated.

Address correspondence to: Roy M. Gulick, Division of Infectious Diseases, Weill Cornell Medicine, Box 125, 1300 York Avenue, New York, New York 10065,
USA. Phone: 212.746.6320; Email: rgulick @med.cornell.edu.

1. Wu Z, McGoogan JM. Characteristics of important lessons from the coronavirus disease 2019 (COVID-19) outbreak in China: Summary of a report of 72314 cases from the Chinese Center for Disease Control Prevention [published online February 24, 2020]. JAMA. https://doi. org/10.1001/jama.2020.2648.

2. CDC COVID-19 Response Team. Severe outcomes among patients with coronavirus disease 2019 (COVID-19) - United States, February 12-March 16, 2020. MMWR Morb Mortal Wkly Rep. 2020;69(12):343-346.

3. Wang $M$, et al. Remdesivir and chloroquine effectively inhibit the recently emerged novel coronavirus (2019-nCoV) in vitro. Cell Res. 2020;30(3):269-271.

4. Goldman JD, et al. Remdesivir for 5 or 10 days in patients with severe COVID-19 [published online May 27, 2020]. N Engl J Med. https://doi. org/10.1056/nejmoa2015301.

5. Liu J, et al. Hydroxychloroquine, a less toxic derivative of chloroquine, is effective in inhibiting SARS-CoV-2 infection in vitro. Cell Discov. 2020;6:16.

6. Gautret P, et al. Hydroxychloroquine and azithromycin as a treatment of COVID-19: results of an open-label non-randomized clinical trial [published online March 20, 2020]. Int J Antimicrob Agents. https://doi.org/10.1016/j.ijantimicag.2020.105949.

7. Geleris J, et al. Observational study of hydroxychloroquine in hospitalized patients with COVID19. N Engl J Med. 2020;382(25):2411-2418.

8. Satlin M, et al. Safety, tolerability, and clinical outcomes of hydroxychloroquine for hospitalized patients with coronavirus 2019 disease. PLoS One. 2020;15(7):e0236778.

9. Tang W, et al. Hydroxychloroquine in patients with mainly mild to moderate coronavirus disease 2019: open label, randomised controlled trial. BMJ. 2020;369:m1849.

10. Joyner MJ, et al. Safety update: COVID-19 convalescent plasma in 20,000 hospitalized patients [published online July 19, 2020]. Mayo Clin Proc. https://doi.org/10.1016/j. mayocp.2020.06.028.

11. Shen C, et al. Treatment of 5 critically ill patients with COVID-19 with convalescent plasma [published online March 27, 2020]. JAMA. https:// doi.org/10.1001/jama.2020.4783.

12. Duan K, et al. Effectiveness of convalescent plasma therapy in severe COVID-19 patients. Proc Natl Acad Sci U S A. 2020;117(17):9490-9496.

13. Mehra MR, Desai SS, Ruschitzka F, Patel AN. RETRACTED: Hydroxychloroquine or chloroquine with or without a macrolide for treatment of COVID-19: a multinational registry analysis. [published online May 22, 2020]. Lancet. https:// doi.org/10.1016/S0140-6736(20)31324-6.

14. Beigel JH, et al. Remdesivir for the treatment of COVID-19 - preliminary report [published online May 22, 2020]. N Engl JMed. https://doi. org/10.1056/NEJMoa2007764.

15. Horby P, et al. Effect of dexamethasone in hospitalized patients with COVID-19: preliminary report [preprint]. Posted on medRxiv June 22, 2020. https://doi.org/10.1101/2020. 06.22.20137273.

16. Cao B, et al. A trial of lopinavir-ritonavir in adults hospitalized with severe COVID-19. $\mathrm{NEnglJ}$ Med. 2020;382(19):1787-1799. 